\title{
Distortion Product Otoacoustic Emissions and Auditory Evoked Potentials in the Hedgehog Tenrec, Echinops telfairi
}

\author{
Markus Drexl, ${ }^{1,4}$ and Michael Faulstich, ${ }^{1,3}, *$ and Boris von Stebut, ${ }^{2, *}$ \\ Susanne RadTKe-Schuller, ${ }^{2}$ AND Manfred Kössl ${ }^{1,4}$ \\ ${ }^{1}$ Zoologisches Institut, Universität München, 80333 München, Germany \\ ${ }^{2}$ Anatomische Anstalt, Universität München, 80336 München, Germany \\ ${ }^{3}$ Salk Institute for Biological Studies, La Jolla, CA 92186, USA \\ ${ }^{4}$ Zoologisches Institut, Universität Frankfurt, 60323 Frankfurt/Main, Germany
}

Received: 23 August 2002; Accepted: 3 July 2003; Online publication: 23 October 2003

\section{ABSTRACT}

The hedgehog tenrec, Echinops telfairi, has certain basal mammalian features, like a cloaca and a sparsely differentiated brain with smooth cerebral hemispheres. The peripheral auditory capabilities of this species were investigated by means of distortion product otoacoustic emissions (DPOAE). For comparison, we determined auditory evoked potentials (AEP) in the inferior colliculus and the auditory cortex. Both methods show that the auditory range of E. telfairi extends well into ultrasonic frequencies, with a region of highest sensitivity at around $16 \mathrm{kHz}$. The total auditory range spans about 4 octaves at $40 \mathrm{~dB}$ SPL. The low-frequency limit of auditory processing is found at frequencies of about $2-3 \mathrm{kHz}$. The DPOAE and the AEP thresholds of E. telfairi do not run fully parallel in the high-frequency range. For a threshold value of $40 \mathrm{~dB}$ SPL, cochlear mechanical thresholds as measured with DPOAE extend up to 50 $\mathrm{kHz}$, whereas neuronal thresholds reach the highfrequency limit at about $30 \mathrm{kHz}$. Frequency tuning, as assessed from DPOAE suppression tuning curves, was low to moderate with $Q_{10 \mathrm{~dB}}$ values ranging from 1.7 to 8 . The lack of discontinuity in the group delay (de-

*Contributed equally to the data presented in this study. Correspondence to: Markus Drexl • Department Biologie II der Ludwig-Maximilians-Universität München - Luisenstr. 14 • 80333 München, Germany - Telephone: + 49-89-5902-202; Fax: +49-69-5902-210; email: markus.drexl@stud.uni-muenchen.de rived from DPOAE measurements) reveals that cochlear frequency representation is tonotopic without any region of specialized mechanical tuning.

Keywords: auditory system, mammal, insectivora, DPOAE, AEP

\section{INTRODUCTION}

Tenrecs are of the order insectivora, which is a rather ancestral group of eutherian mammals. It is argued that insectivores have retained primitive features and thus are closer to the ancestral stock than species of other living mammalian orders (see Springer et al. 1997), and that the study of insectivora brain organization provides insight into the brain of early ancestors of eutherian mammals (Stephan et al. 1991).

Besides advanced characteristics, tenrecs have retained conservative physiological and morphological features, e.g., an unstable thermoregulation and a reduced visual sense, the retention of testes within the body cavity, and a cloaca (Gould 1965; Eisenberg and Gould 1970). The encephalization index of tenrecs is the least among extant mammals, with a sizable bulbus olfactorius and smooth cerebral hemispheres (Stephan et al. 1991). The forebrain of tenrecs closely resembles that of early mammals in relative size and small neocortex (Künzle and Rehkämper 1992; Krubitzer et al. 1997). These features lead to the 
classification of tenrecs as primitive eutherian mammals.

Tenrecs are confined to Madagascar. They become active at dusk and hunt during the night. They search for insects on the ground and in the branches of trees and shrubbery (Eisenberg and Gould 1970). This lifestyle requires reliable orientation in the dark. Gould (1965) demonstrated the importance of hearing. Furthermore, he suggested that tenrecs are even capable of a crude mode of echolocation. They emit short clicks produced by tongue and lip movements, a technique similar to that utilized by the fruit bat Rousettus aegyptiacus, one of the few megachiropteran bats capable of echolocation (see Koay et al. 1998). These broadband calls, that cover a frequency range from about 5 to $17 \mathrm{kHz}$, may improve the nearfield orientation of $E$. telfairi the night. Unfortunately, to our knowledge there exists no systematic study on the properties of the middle ear and the cochlea of this species.

We investigated the hearing capability of E. telfairi by determining the mechanical frequency processing of the inner ear by means of cochlear distortions. These frequency distortions are produced by a nonlinear amplification process of the inner ear (see, e.g., Dallos 1992) and are propagated back to the tympanum where they can be measured with a sensitive transducer. The distortion product otoacoustic emissions (DPOAE) have become a common tool in hearing research. They reflect general properties of a subject's peripheral auditory processing (Probst et al. 1991; Whitehead et al. 1996; Shera et al. 1998) and can be used to predict auditory thresholds (Boege et al. 2002; Gorga et al. 1997). DPOAE-based auditory threshold curves usually correlate well with behavioral thresholds (Faulstich et al. 1996; Faulstich and Kössl 2000) We determined DPOAE thresholds of the relative sensitivity of nonlinear cochlear mechanics by presenting two pure tones of the primary frequencies $f_{1}$ and $f_{2}$ and measuring the most prominent cubic distortion product $2 f_{1}-f_{2}$. Mechanical tuning properties of the cochlea were derived from isosuppression tuning curves (STC).

Central processing of auditory information was investigated by recording auditory evoked potentials (AEP) in the inferior colliculus (IC) and in the auditory cortex (AC). Almost all auditory information converges in the midbrain auditory center, the IC, with extensive afferent projections to higher and lower auditory nuclei as well as to the motor system (Casseday and Covey 1996). It plays a direct or indirect role in virtually all sound-related behavior. The IC of different species has been the subject of many studies on central processing of auditory information and is well suited for an interspecies comparison. The neural thresholds of the AC give further information on central auditory processing. The investigation of the auditory capabilities of E. telfairi as a "basal" mammal should allow the assessment of evolutionary development of hearing in other eutheria and provide a basis for comparison with Monotremata and Marsupialia.

\section{MATERIAL AND METHODS}

A total of 19 adult lesser hedgehog tenrecs (E. telfairi) of both sexes were used in this study. The animals were taken from the breeding colony of H. Künzle (Künzle 1998). Animal weight varied from 70 to 185 $\mathrm{g}$. This wide range of body weight is related to the seasonal activity pattern of E. telfairi, which undergoes a torpor period from about October to March in this colony. Anesthesia was initiated with Ethrane ${ }^{\circledR}$ (by inhalation). During experimentation, the animals were anesthetized with Tribromethanol (TBE, 1.0 $\mathrm{ml} / 100 \mathrm{~g} \mathrm{BW}, \mathrm{IP})$. The experiments were performed in a sound-attenuated chamber.

\section{Otoacoustic emission measurements}

DPOAE measurements focused on 11 animals. Anesthesia was necessary to reduce animal movements and thus the risk of tympanic membrane injury caused by the acoustic coupler. A single recording session lasted up to $3 \mathrm{~h}$. A homeothermic blanket was used to maintain the body temperature at about $29^{\circ} \mathrm{C}$. The animal's head was fixed with a mouth holder. A closed coupler system, containing three $(0.5$-in. B\&K 4133 microphones that served as loudspeakers and one recording microphone (0.25-in. B\&K 4135), was positioned in the animal's meatus close to the eardrum. Two of the speakers were used to transmit the pure-tone stimuli $\left(f_{1}\right.$ and $\left.f_{2}\right)$. The third optional speaker was used to present a suppressor tone $\left(f_{3}\right)$ in the course of suppression tuning curve measurements (STC). A detailed description of the acoustic coupler system is given by Kössl (1994).

Stimulus generation and data acquisition were controlled by Testpoint programs (Keithley, Cleveland, $\mathrm{OH})$. The pure-tone stimuli $\left(f_{1}, f_{2}\right.$, and optionally $f_{3}$ ) were generated on two Microstar (Bellvue, WA) DAP digital signal-processing boards, D/A-converted, and fed to two or three, respectively, GPIBcontrolled attenuators (designed by Jim Hartley, University of Sussex, Brighton), finally amplified and transmitted via the loudspeakers.

The speakers were separately calibrated in situ for frequencies between 1 and $100 \mathrm{kHz}$. If necessary, these acoustic measurements were repeated during the experiment (following animal head movements). The measured signals were amplified and fed into the 
A/D input of the DAP boards. The input and output channels of the board were sampled synchronously at $333 \mathrm{kHz}$ per channel. The two primary stimuli $f_{1}$ and $f_{2}$ were presented at a starting phase of $0^{\circ}$. The starting phase of $f_{3}$ was not fixed.

\section{Distortion threshold curves}

DPOAEs are elicited by the interaction of two traveling waves on the basilar membrane induced by the simultaneous presentation of two pure-tone stimuli of different frequency $\left(f_{1}\right.$ and $\left.f_{2}\right)$. In the zone of overlap both oscillations are amplified in a nonlinear way, thus producing frequency distortions. The place of distortion generation in the cochlea appears to be close to the frequency place of $f_{2}\left(f_{1}<f_{2}\right.$; Brown and Kemp 1984). Consequently, we plotted the distortion threshold curve as a function of $f_{2}$. DPOAE threshold curves were measured for $f_{2}$ frequencies from 5 to 70 kHz. By keeping $f_{2}$ constant and varying $f_{1}$ the optimum stimulus separation (best ratio $f_{2} / f_{1}$ ) was determined, which produced maximum DPOAE levels at low stimulus levels. With the primary tones set at best ratio, growth functions of the $2 f_{1}-f_{2}$ distortion were measured by stepwise increasing the stimulus levels. The level of $f_{2}\left(l_{2}\right)$ was set to the level of $f_{1}\left(l_{1}\right)$ $-10 \mathrm{~dB}$, a stimulus condition proven to produce high DPOAE levels in mammals (Brown and Kemp 1984; Probst et al. 1991; Mills 1997). The level of $f_{2}$ sufficient to elicit a DPOAE of $-10 \mathrm{~dB}$ SPL was defined as threshold criterion. Isodistortion threshold curves reflect auditory sensitivity in mammals as well as in reptiles (Kössl 1992, 1994; Manley et al. 1993; Faulstich et al. 1996; Faulstich and Kössl 1999).

\section{DPOAE suppression tuning curves}

Cochlear tuning properties were obtained by recording DPOAE suppression tuning curves (STC). After adjusting $f_{1}$ to the best ratio $f_{2} / f_{1}$, stimuli levels were chosen to elicit a $2 f_{1}-f_{2}$ DPOAE between -5 to $5 \mathrm{~dB}$ SPL. At these levels, DPOAEs were more than 6 $\mathrm{dB}$ above the noise level and were sufficiently robust for suppression experiments. A pure-tone $f_{3}$, which was not phase fixed with respect to the stimuli, was presented simultaneously with the $f_{1}$ and $f_{2}$ stimuli via the third loudspeaker to suppress the DPOAE. For each primary stimulus pair, a large range of $f_{3}$ frequencies was tested. The $f_{3}$ level was increased in $5 \mathrm{~dB}$ steps and the DPOAE level was measured. The $f_{3}$ level sufficient to reduce the OAE by $6 \mathrm{~dB}$ was plotted as a function of the $f_{3}$ frequency to derive the tuning curve (e.g., Brown and Kemp 1984; Martin et al. 1987). The separation of $f_{3}$ from the stimulus frequencies and to the DPOAE frequency was at least $200 \mathrm{~Hz}$ to avoid direct interaction of the tones. From the STC the best frequency $(\mathrm{BF})$, i.e., the frequency of the absolute minimum of the STC, the threshold at $\mathrm{BF}$, and the $Q_{10 \mathrm{~dB}}$ were determined.

\section{DPOAE group delay}

Evoked otoacoustic emissions have a certain latency relative to the onset of the stimuli. In the case of DPOAEs, this latency is thought to depend on the cochlear traveling times and on the delay of the distortion generation. It is difficult to determine the latency from measurements in the time domain since stimuli and DPOAEs largely overlap. However, the phase of the DPOAE can be used to measure the ongoing transmission delay of DPOAEs. Specifically, the gradient of DPOAE phase $(p h)$ versus DPOAE frequency $(f)$ defines the group delay, which is the ongoing delay of the main emission wave front (e.g., Kimberley et al. 1993). The magnitude of the group delay of DPOAEs is a measure of the travel time and depends on the stimulus paradigm used (O'Mahoney and Kemp 1995; Whitehead et al. 1996). To gain information on cochlear traveling times and under the assumption that the $2 f_{1}-f_{2}$ distortion is generated at the $f_{2}$ representation site (e.g., Brown and Kemp 1984), the frequency of $f_{2}$ was fixed and $f_{1}$ was shifted to lower frequencies. The corresponding level and the phase of the $2 f_{1}-f_{2}$ DPOAE were measured for different $f_{2}$ frequencies and at a number of stimulus levels. The measured phase of the DPOAE was corrected for the stimulus phases according to Mills and Rubel (1997). After unrolling phase transitions from $-180^{\circ}$ to $+180^{\circ}$ by subtracting $360^{\circ}$, the mean group delay $\left(-\Delta p h / \Delta f * 360^{\circ}\right)$ was calculated over the frequency range for which the DPOAE level reached its maximum. The group delay values were plotted as a function of $f_{2}$.

\section{Neurophysiological recording}

We recorded AEPs of E. telfairi in eight specimens over a stimulus frequency range of $2-80 \mathrm{kHz}$. For surgery, the animal's head was stabilized with a mouth holder and two ear pins. The pedal withdrawal reflex served as a measure for the state of anesthesia. An incision along the midsaggital plane of the skull was made and the tissue was pushed laterally with a retractor. A metal pin was glued to the midline of the skull with dental cement. This pin served to attach the animal to the stereotactic device (see Schuller et al. 1986). When the animal was placed in the experimental setup, its position was aligned along three axes to achieve a standard position. A reference point on the surface of the skull (hemispheric divergence, hd) was defined. The penetration point was located 
relative to this mark. For each electrode penetration, a small hole was drilled into the skull.

Tungsten electrodes were used for AEP recording (impedance $<2 \mathrm{M} \Omega$ ). The signal was amplified [Bioamp Headstage HS4; Tucker Davis Technologies (TDT), Gainesville, FL, Bioamp controller DB4], band pass filtered (TDT Bioamp controller DB4; Spike Conditioner PC1), monitored on an oscilloscope (Hameg HM 1007), and fed via a custom-made interface to a PC for analysis. Usually 20 passes per stimulus presentation were averaged.

Pure-tone stimuli were used to determine the neural response. The presentation interval was 500 $\mathrm{ms}$ and the stimulus duration was $200 \mathrm{~ms}$. The rise/ fall time was set to $3 \mathrm{~ms}$. The stimuli were generated on an array processor card (TDT AP2), D/A-converted (TDT DA3-2), and fed to an anti-aliasing filter (TDT FT5). The stimulus level was set with two programmable attenuators (TDT PA4) connected in series. The stimuli were amplified (Rotel Power Amplifier RB 951, Rotel, North Reading, MA) and transmitted to the calibrated loudspeaker, which was positioned at a distance of $25 \mathrm{~cm}$, aimed to the center of the pinna at an angle of $45^{\circ}$ contralateral to the neurophysiological recording side. The sound system was calibrated in the recording setup, where a 0.25-in. B\&K microphone was placed instead of the animal at the position of the tympanum to record the frequency response of the loudspeakers. The frequency response of the used speaker was flat within $\pm 10 \mathrm{~dB}$ for frequencies between 10 to $80 \mathrm{kHz}$. Harmonic distortions of the speaker for the stimulus frequencies used were at least $40 \mathrm{~dB}$ below the level of the pure-tone stimulus and therefore did not produce artefacts. Stimulus presentation, signal recording, and averaging were controlled by a timing generator (TDT TG6). The threshold of the AEP was determined as that stimulation level at which the averaged neuronal response was no longer distinguishable from the noise floor. The level resolution was set to $5 \mathrm{~dB}$.

\section{RESULTS}

\section{Distortion threshold curves}

The distortion thresholds were determined for $f_{2}$ frequencies from 5 to $70 \mathrm{kHz}$ (Fig. 1). The best frequency ratios $\left(f_{2} / f_{1}\right)$ resulting in highest levels of $2 f_{1}-f_{2}$ ranged from 1.11 to 1.29 . No significant correlation between the best frequency ratio and $f_{2}$ could be found. The threshold curve of E. telfairi is broadly tuned with a shallow low-frequency and a steeper high-frequency flank. It ranges from about 5 to 50 $\mathrm{kHz}$ (at $40 \mathrm{~dB}$ SPL). Below $5 \mathrm{kHz}$ no DPOAE could be elicited, even with primary $f_{2}$ levels of $70 \mathrm{~dB}$ SPL. The

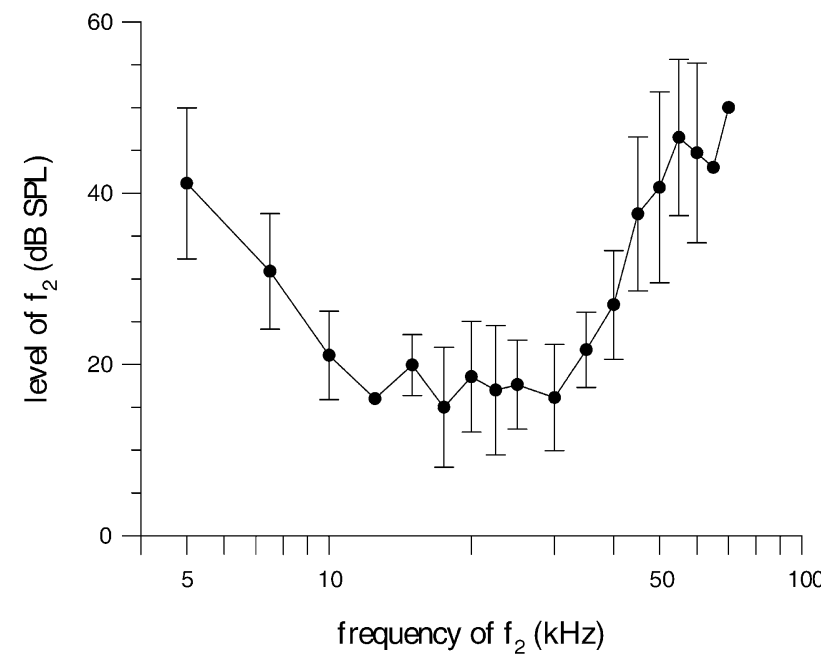

FIG. 1. DPOAE threshold curve from 5 to $70 \mathrm{kHz}$ for $E$. telfairi. The level of $f_{2}\left(I_{2} ; I_{2}=I_{1}-10 \mathrm{~dB}\right)$ sufficient to generate a $2 f_{1}-f_{2}$ DPOAE of $-10 \mathrm{~dB} S P L$ is plotted as a function of the $f_{2}$ frequency. The best frequency ratios $\left(f_{2} / f_{1}\right)$ resulting in highest levels of $2 f_{1}-f_{2}$ ranged from 1.11 to 1.29 . No significant correlation between the best frequency ratio and $f_{2}$ could be found. Means (filled circles) and standard deviations (error bars) are shown.

minimum of the average distortion threshold was between 10 and $30 \mathrm{kHz}$ at $f_{2}$ levels of approximately $20 \mathrm{~dB}$ SPL. The large variability of the threshold data may partly be caused by physiological changes that are linked to variations of general activity of the animal.

\section{Suppression tuning curves}

The isosuppression tuning curves (STC) measured in the tenrec display an almost symmetrical shape with the low-frequency flank only slightly shallower than the high-frequency flank. Frequency tuning sharpness was low to moderate with $Q_{10 \mathrm{~dB}}$ values ranging from 1.7 to 8 . The minimum was close to $f_{2}$ for all STCs at $f_{2}<30 \mathrm{kHz}$ (Fig. 2), suggesting that the generation of DPOAE takes place near the $f_{2}$ place on the basilar membrane like in other mammals (e.g., Brown and Kemp 1984). For $f_{2}>30 \mathrm{kHz}$, the minimum of the STCs determined in one individual did not correlate with $f_{2}$ anymore but stayed around 30$35 \mathrm{kHz}$ (Fig. 2f). This was also found in the opossum for $f_{2}$ frequencies around $45 \mathrm{kHz}$ (Faulstich et al. 1996). The best frequency ratios $\left(f_{2} / f_{1}\right)$ resulting in highest levels of $2 f_{1}-f_{2}$ ranged from 1.11 to 1.33 in this animal. There was no correlation between the best frequency ratio and the $Q_{10 \mathrm{~dB}}$ value.

\section{Group delay}

When $f_{2}$ is held constant and $f_{1}$ is lowered in small steps, $2 f_{1}-f_{2}$ DPOAEs can be measured for a wide 


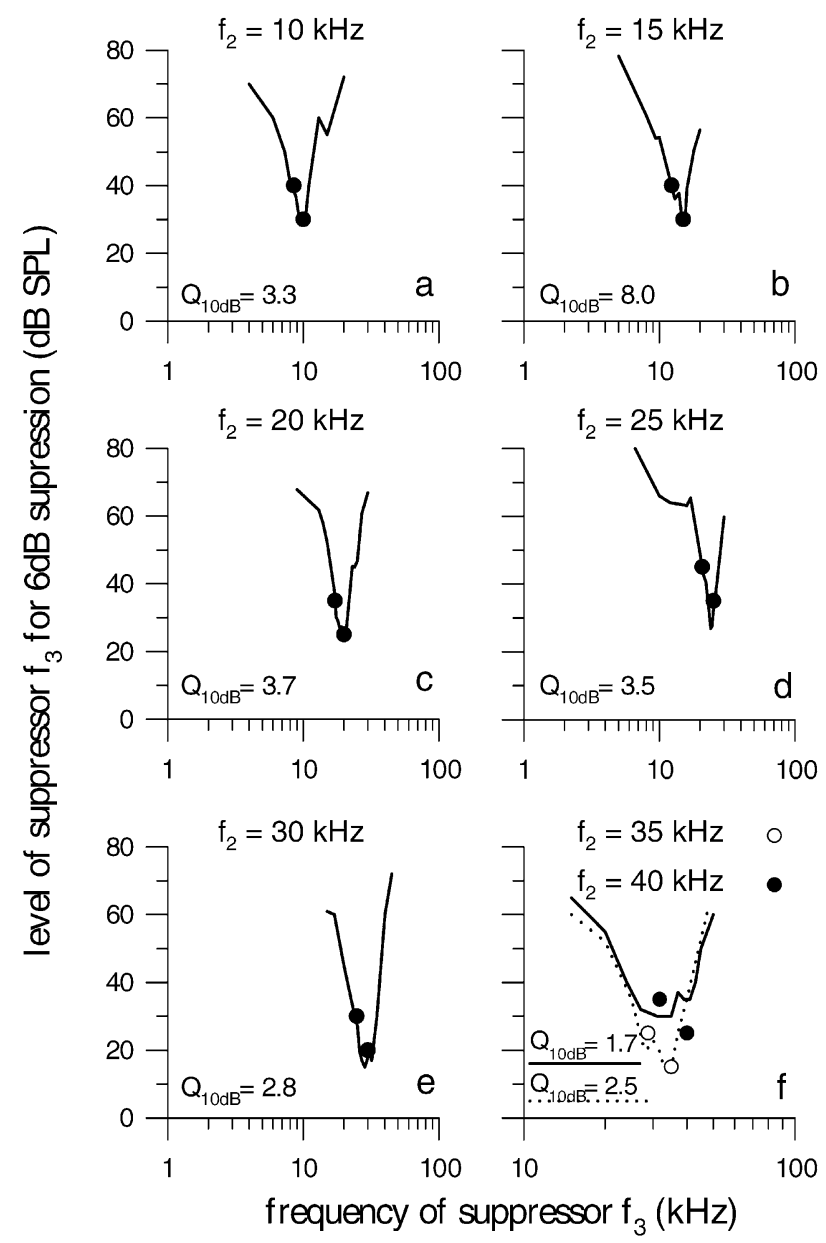

FIG. 2. DPOAE isosuppression tuning curves from one animal. The level of $f_{3}$ necessary to suppress $2 f_{1}-f_{2}$ by $6 \mathrm{~dB}$ is plotted as a function of the frequency of $f_{3}$. Circles represent the frequency and level of the primaries. The STC's $Q_{10 d B}$ values are shown in the lower left of each panel. For $f_{2}$ frequencies greater than $30 \mathrm{kHz}$, the minimum of the STCs no longer follows the primary frequencies. The $f_{2}$ frequency is indicated at the top of the corresponding panel. The best frequency ratios $\left(f_{2} / f_{1}\right)$ resulting in highest levels of $2 f_{1}-f_{2}$ ranged from 1.11 to 1.33 . There was no correlation between the best frequency ratio and the $Q_{10 \mathrm{~dB}}$ value. The level of $f_{2}$ was set to the level of $f_{1}-10 \mathrm{~dB}$.

range of $f_{1}$ frequencies. The DPOAE phase changes regularly with each $f_{1}$ step and a rather constant group delay value results, indicating the slope of the phase gradient. The group delay was averaged over as many points as possible (at least 15 points) for which a continuous phase was present (for a detailed methodological description, see Faulstich and Kössl 2000).

The group delay was measured in a single individual of E. telfairi where large distortions could be elicited over a wide frequency range. The stimulus level was at least $6 \mathrm{~dB}$ above distortion threshold levels. The mean group delay decreased with increasing $f_{2}$. It was highest $(2.23 \mathrm{~ms})$ for $f_{2}=5 \mathrm{kHz}$ and drops to a minimum of $0.17 \mathrm{~ms}$ for $f_{2}=40 \mathrm{kHz}$. The

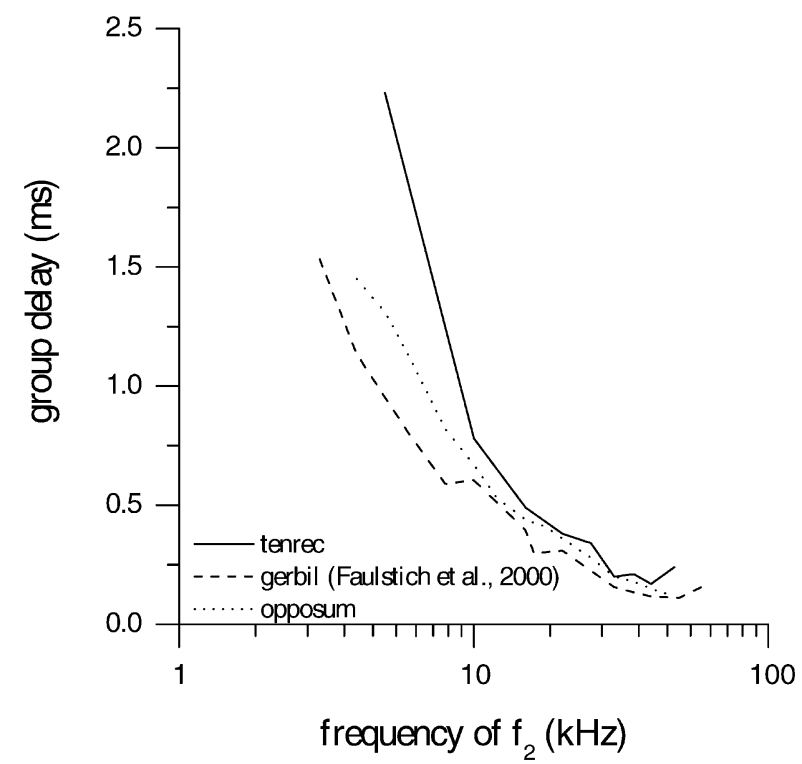

FIG. 3. The group delay (ms) of the distortion product as a function of frequency $(\mathrm{kHz})$ of the tenrec (solid line). For comparison, data from the gerbil (dashed line) and the opossum (dotted line) are shown. To determine the group delay, $f_{2}$ was held constant while $f_{1}$ was decreased in small steps. For each $f_{1}$ step, the $2 f_{1}-f_{2}$ DPOAE level and its phase was determined as a function of the $2 f_{1}-f_{2}$ frequency. Fromthe change in DPOAE phase relative to the change in frequency, the mean group delay values were calculated over all values for which the ratio curve was at least $6 \mathrm{~dB}$ above noise floor.

course of the mean group delay is similar to those measured in the gerbil and the opossum with the same experimental setup (Fig. 3).

\section{Neurophysiological thresholds}

Auditory evoked potentials from a total of 22 electrode penetrations in eight animals were analyzed to determine the neurophysiological threshold of E. telfairi in the IC. In the presumed AC, 24 electrode penetrations in six animals were conducted. Frequencies from 2 to $80 \mathrm{kHz}$ were tested comprising $E$. telfairi's hearing range as determined in the preceding otoacoustic experiments. The penetration loci in the IC were distributed over the whole nucleus including rostral, central, caudal, and lateral parts (see Fig. 4a) at different penetration depths (covering the whole dorsoventral extension of the IC of $3000 \mu \mathrm{m}$ ). The shape as well as the absolute sensitivity of individual EP thresholds was rather similar across penetrations, indicating that the electrodes used did not locally discriminate and yielded a gross response covering the entire IC (Fig. 4b). Control measurements adjacent to the IC did not elicit responses to auditory stimuli.

Similar to the threshold determined by DPOAE measurements, the neurophysiological response extends to ultrasonic frequencies (Fig. 5). The range of 


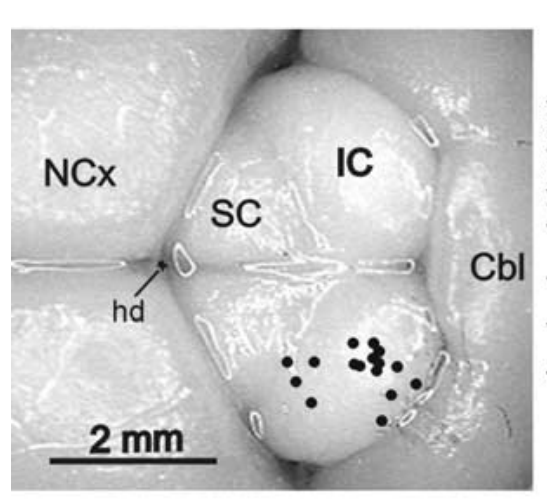

a

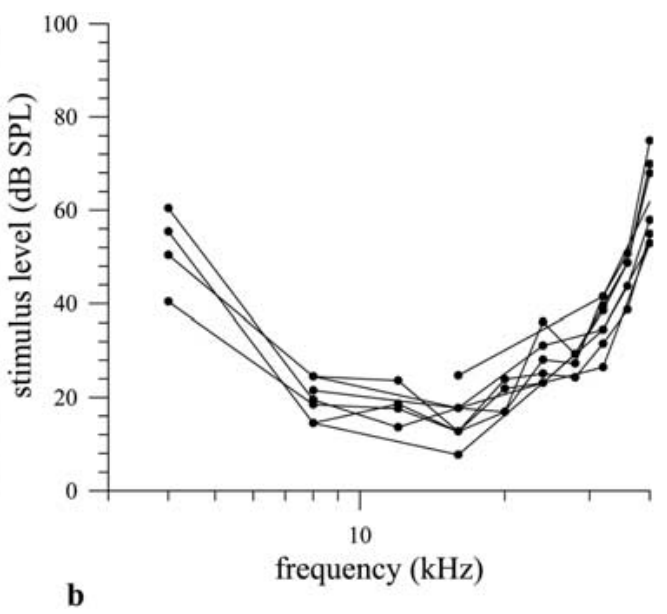

FIG. 4. Evoked potential recording in the IC. a IC-recording positions. The penetration sites across individuals were normalized according to two references; Cbl-Cerebellum,

IC-Colliculus inferior, $\mathrm{NCx}$-Neocortex, SC-Colliculus superior, hd-hemispheric divergence. b Examples of audiograms from separated loci across the IC in one animal. Regardless of the penetration site, the course of the threshold curves evoked by acoustic stimulation is similar.

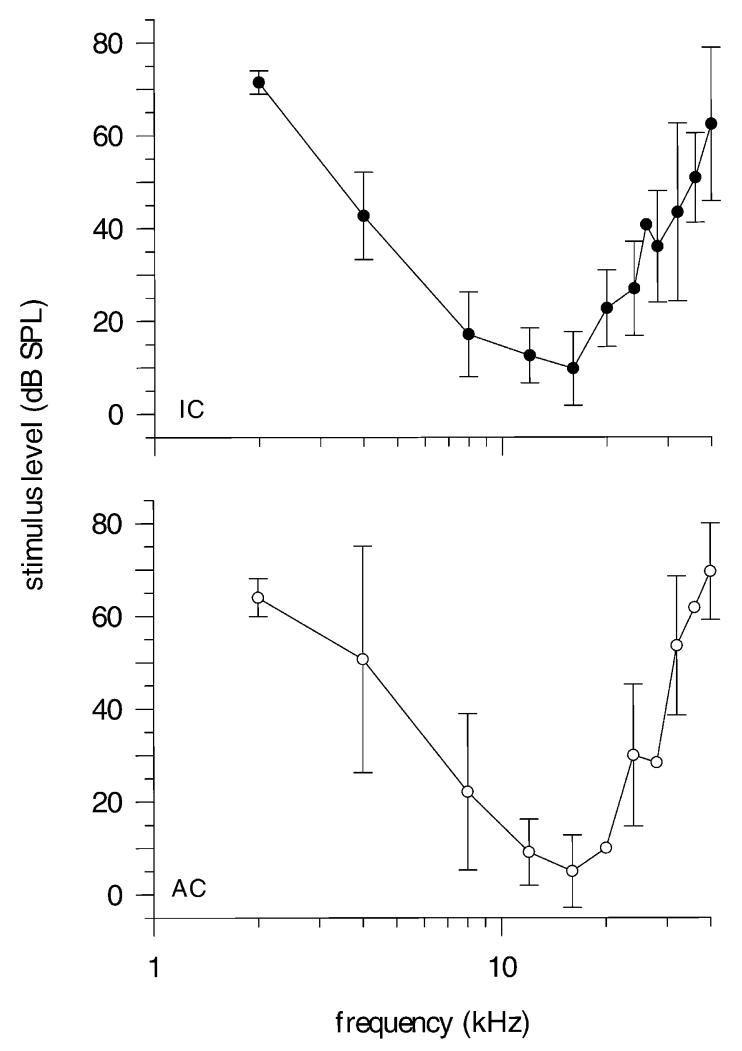

FIG. 5. Neurophysiological IC (filled circles) and AC (open circles) audiograms from 2 to $40 \mathrm{kHz}$ in E. telfairi. Means (circles) and standard deviations (error bars) are shown.

best sensitivity both in the IC and the AC is found between 8 and $20 \mathrm{kHz}$ with a threshold minimum of about $10 \mathrm{~dB}$ SPL (IC) or $5 \mathrm{~dB}$ SPL (AC), respectively, at $16 \mathrm{kHz}$. In comparison to the DPOAE data, the threshold curve of AEP is slightly shifted to lower frequencies. The lower-frequency range measured with AEPs extends to $2-3 \mathrm{kHz}$ in comparison to $5 \mathrm{kHz}$ for the DPOAE thresholds. For frequencies above 20 $\mathrm{kHz}$, the neurophysiological threshold consistently increases, reaching the upper limit at about $30 \mathrm{kHz}$ (at $40 \mathrm{~dB}$ SPL). Thus, IC and AC thresholds are tuned to lower frequencies and the distortion product threshold is more broadly tuned than the neurophysiological response.

\section{DISCUSSION}

The auditory range of E. telfairi extends to ultrasonic frequencies with a broad threshold minimum. The frequency range of this region differs, depending on whether the ascending auditory pathway was investigated at a peripheral or a central position. The DPOAE threshold curve is plotted as a function of $f_{2}$ frequency since the cubic distortions products, which provide the basis for the threshold curves, are generated close to the $f_{2}$ frequency place (e.g., Brown and Kemp 1984). It reveals a frequency range of minimum thresholds between 10 and $35 \mathrm{kHz}$ (Fig. 1). The associated $f_{2}$ level sufficient to elicit distortions is slightly below $20 \mathrm{~dB}$ SPL. The threshold ranges from about 5 to $50 \mathrm{kHz}$ (at $40 \mathrm{~dB}$ SPL).

The thresholds based on AEPs, recorded in the IC and the AC, reveal a low-frequency limit of 2-3 kHz. At frequencies above $25 \mathrm{kHz}$, the neuronal auditory sensitivity decreases steeply with increasing frequency (Fig. 6). The differences among otoacoustic and neurophysiological thresholds at low frequencies are also found in other mammals, e.g., in the gerbil Meriones unguiculatus (Faulstich and Kössl 2000), in the rhesus monkey Macaca mulatta (Lasky et al. 1999), in the Echidna (Tachyglossus aculeatus; Mills and Shepherd 2001), and in the opposum (Monodelphis domestica; Faulstich et al. 1996). In these species the course of neurophysiologically measured threshold curves extends to lower frequencies than that of DPOAE thresholds (see also Fig. 7). In general, differences between DPOAE measurements made with a closed system and free-field neurophysiological data 


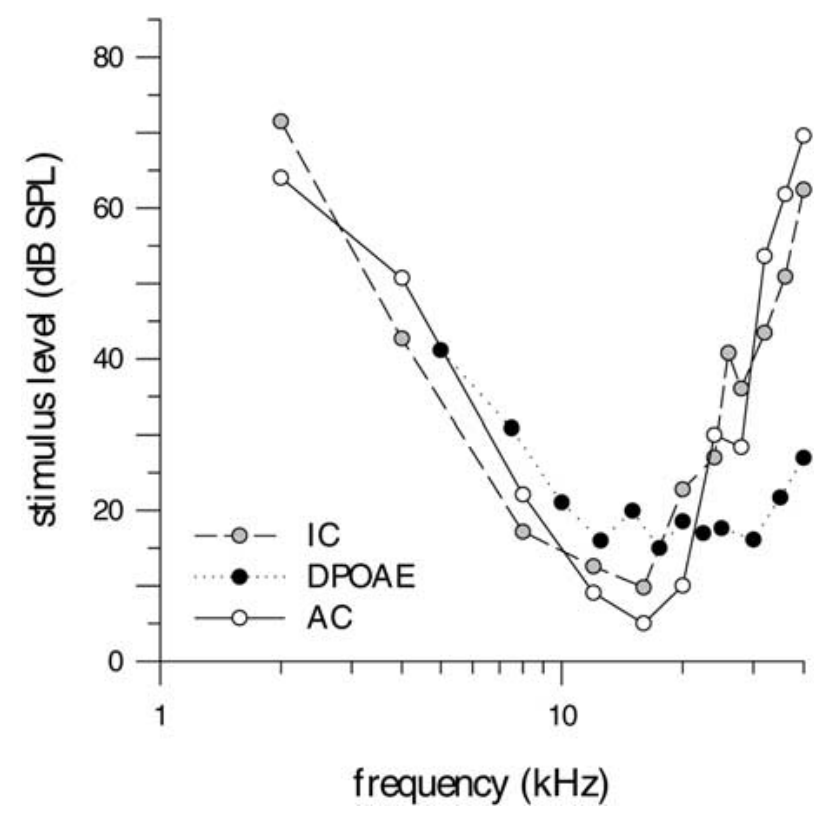

FIG. 6. Neurophysiological IC (gray circles) and AC (open circles) audiograms from 2 to $40 \mathrm{kHz}$ in relation to the DPOAE audiogram (filled circles) of E. telfairi.

might be caused by a notch in the head related transfer function (HRTF) or an antiresonance in the free-field system. Nevertheless, neurophysiological and DPOAE data from the Echidna (Fig. 7) were both obtained under closed-field conditions, but still reveal differences comparable to the ones found in the tenrec, suggesting that the arguments given above might contribute to these differences, but may not be the sole source. Differences between DPOAE and neurophysiological data seem to be a general feature found in a wide range of mammals and suggest an intrinsic difference between neuronal and otoacoustic emission responses, which might be related to the suggestion that DPOAEs reflect the activity of the cochlear amplifier, whereas neuronal threshold curves are further shaped by additional processes in the ascending auditory pathway. When assessing the difference between DPOAE and neurophysiological data, one needs to keep in mind that the middle ear has more influence on the DPOAE than on neurophysiological measures. In the gerbil, it may act as a high-pass filter, thus altering the measurable distortion product in the ear canal (Faulstich and Kössl 1999). However, since E. telfairi is much less sensitive for low frequencies than the gerbil, middle-ear highpass filtering seems to be less important. The neurophysiological range is shifted toward lower frequencies with an area of highest sensitivity between 10 and $16 \mathrm{kHz}$. In addition to the suggestion made above, this might also be related to the auditory gain originating from the pinna, since it is known that in other species, depending on the size of the pinna, amplifi- cation effects are most prominent at frequencies ranging from about 2.5 to $30 \mathrm{kHz}$ (e.g., Murphy and Davis 1998; Guppy and Coles 1988; Coles and Guppy 1986; Jen and Chen 1988) and can amount to values as much as $20 \mathrm{~dB}$. As the DPOAEs were measured directly at the eardrum, pinna effects are excluded in these measurements. The neurophysiological data reveals an upper limit of about $30 \mathrm{kHz}$ (at $40 \mathrm{~dB}$ SPL), associated with a sharp high-frequency cutoff, corresponding to more than $30 \mathrm{~dB} /$ octave. Despite the relatively high standard deviations, the AEP highfrequency threshold is up to $30 \mathrm{~dB}$ above that of the concomitant DPOAE threshold.

The peripheral tuning, determined by isosuppression tuning curves (STC), displays an almost symmetrical shape. $Q_{10 \mathrm{~dB}}$ values of $1.7-8$ reveal a low to moderate frequency tuning (Fig. 2). The minimum was close to $f_{2}$ for frequencies below $30 \mathrm{kHz}$. Consequently, like in other mammals, the distortion generation most probably took place near the $f_{2}$ position. For frequencies above $30 \mathrm{kHz}$, the minimum in STC was not correlated to $f_{2}$ but stayed around $30-35 \mathrm{kHz}$ (Fig. 2f). The same response characteristics were also found in the opossum Monodelphis domestica at $f_{2}$ frequencies around $45 \mathrm{kHz}$ and may indicate the end of frequency representation in the cochlea (Faulstich et al. 1996), which is further supported by the steep increase of the distortion threshold at high frequencies.

Evoked otoacoustic emissions have a certain latency relative to the onset of the stimuli. In the case of DPOAEs, this latency is thought to depend on the cochlear traveling times and on the delay of the distortion generation. Thus, the calculation of the group delay, expressed as the gradient of DPOAE phase versus DPOAE frequency, can give information about the peripheral frequency representation and specializations in cochlear mechanics.

The mean group delay of E. telfairi decreases with increasing $f_{2}$ from $2.23 \mathrm{~ms}$ at $5 \mathrm{kHz}$ to $0.17 \mathrm{~ms}$ at 40 $\mathrm{kHz}$ (Fig. 3). The course of the mean group delay (Fig. 3) is similar to those of the gerbil (Faulstich and Kössl 2000) and the opossum. The minimum values are almost identical for the three species for $f_{2}$ frequencies greater than $10 \mathrm{kHz}$. For lower frequencies, the mean group delay reaches similar values for different frequencies and seems to be correlated to the lower flank of the threshold. The lack of discontinuity in the group delay reveals that the peripheral frequency representation is tonotopic without any region of enhanced mechanical tuning.

Both the range and the shape of the threshold of E. telfairi correspond well to the typical mammalian pattern. Nevertheless, the comparison within eutherian mammals reveals differences in low-frequency auditory processing. When investigating the low-fre- 

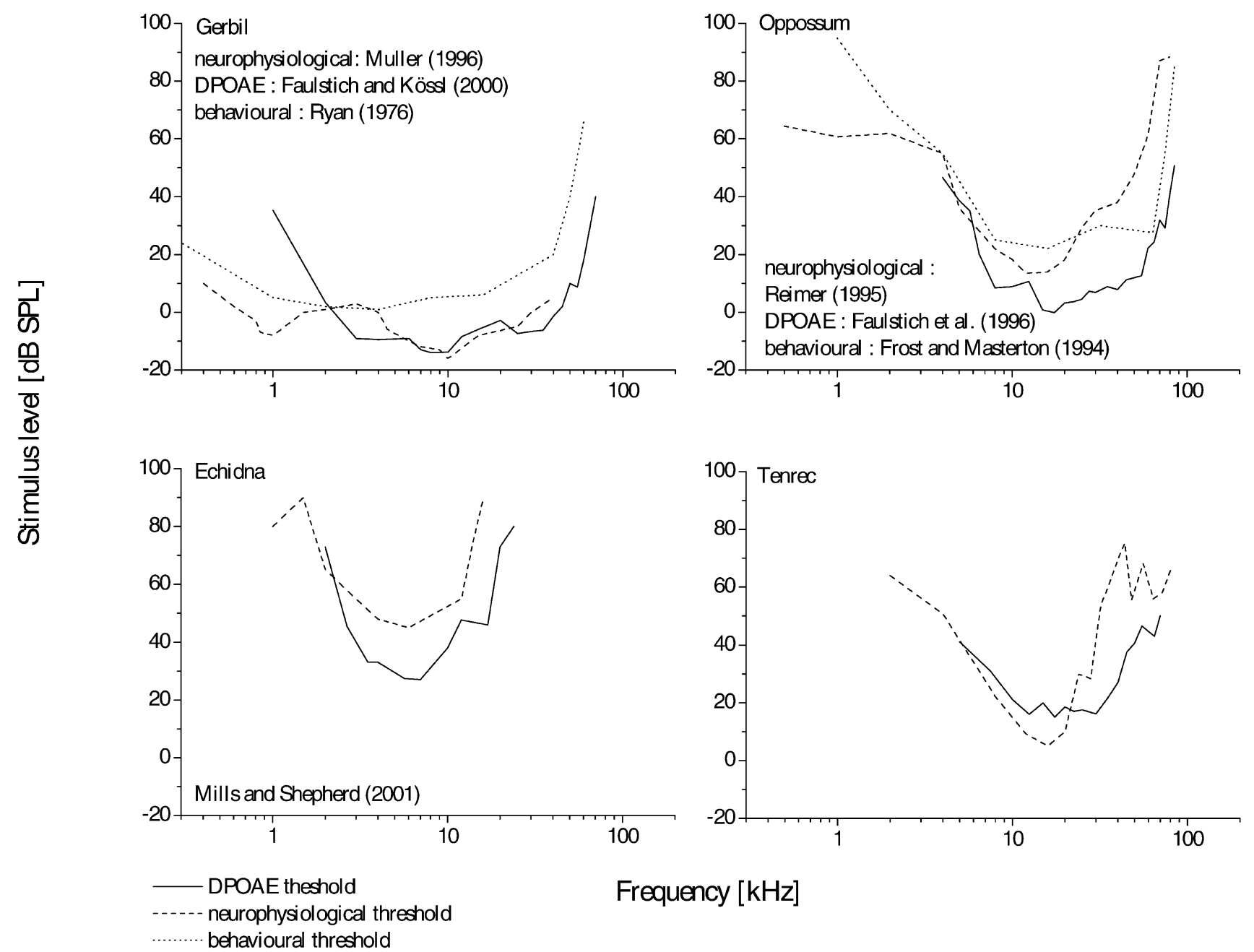

Frequency $[\mathrm{kHz}]$

FIG. 7. Comparison of DPOAE (solid line) and neuronal (dashed line) thresholds from the tenrec, gerbil, opossum, and echidna. In addition, behavioral threshold curves are shown for the gerbil and opposum (dotted line).

quency limit of 58 mammalian species, Heffner et al. (2001) found a bimodal distribution with species sensitive to frequencies below $125 \mathrm{~Hz}$ being separated from those with a low-frequency limit above $500 \mathrm{~Hz}$. Nevertheless, there is no obvious morphologic, phylogenetic or ecological parameter that allows a distinction between members of these two groups. A similar divergence is found in rodents. Restricted lowfrequency processing is found, for example, in the spiny mouse (Acomys cahirinus), whereas the chipmunk (Tamias striatus), the groundhog (Marmota monax), the hamster (Mesocricetus auritus), the chinchilla (Chinchilla chinchilla), and the gerbil (Meriones unguiculatis) belong to the "low-frequency" group (Heffner and Heffner 1991; Faulstich and Kössl 1999; Heffner et al. 2001). The low-frequency limit apparently depends on features that are not primarily related to a species phylogeny. Anatomical investigations of the cochlea indicate that, concerning its acoustic sense, E. telfairi is a generalist without any obvious specializations (M. Vater, personal communication).

When compared with eutherians, marsupials are still considered "primitive," although they successfully adapted to a wide range of different lifestyles. These adaptations certainly include the auditory system. The middle ear and the inner ear as well as the neuronal organization and connectivity were studied in representatives and found to be rather similar to those of eutherians (Willard and Martin 1983; Muller et al. 1993; Aitkin 1995). The hearing capabilities of the echidna, Tachyglossus aculaetus (Monotremata), are found to be midway between mammals and birds (Mills and Shepherd 2001). This species resembles $E$. telfairi in both appearance and lifestyle. Tachyglosssus' internal temperature is relatively low $\left(<32^{\circ} \mathrm{C}\right)$ for mammals, and it possesses a cloaca. Its acoustic sensitivity, investigated by DPOAE and auditory brainstem responses, is best in a frequency range of 4-8 $\mathrm{kHz}$ and the absolute threshold values are compar- 
able to that of eutherian mammals (Fig. 7). However, the effective auditory range comprises only 3 octaves, and the high-frequency limit of $T$. aculaetus is restricted to frequencies unusually low for mammals. The auditory range of $E$. telfairi with a high-frequency limit of $40 \mathrm{kHz}$ and a frequency range of only 4 octaves resembles that of $T$. aculaetus. Mammals typically are able to perceive frequencies up to $30-50 \mathrm{kHz}$. Specialists extend this range to beyond $100 \mathrm{kHz}$. Even in birds with a specialized auditory system, the hearing range is restricted to frequencies below $12 \mathrm{kHz}$ (for review, see Manley 1990).

Variations in high-frequency hearing have been attributed to the evolutionary pressure involving sound localization, as a species' high-frequency hearing limit varies inversely with interaural distance. Mammals with small heads and ears need to hear higher frequencies to use interaural intensity differences and pinna cues for sound localization than larger mammals (Heffner et al. 2001). Early mammals are supposed to have been insectivore-like, with small heads and a good high-frequency hearing (Popper and Fay 1997). Apparently, E. telfairi, with an auditory threshold comprising about 4 octaves, including restricted low-frequency hearing, a high-frequency limit at about $40 \mathrm{kHz}$, and a moderate sensitivity in the range of best hearing between about 10 and $16 \mathrm{kHz}$, complies with eutherian ancestors.

\section{ACKNOWLEDGMENTS}

H. Künzle placed tenrecs of his breeding colony at our disposal. The neurophysiological experiments were conducted in his laboratory. G. Schuller contributed during the startup to the design and realization of the neurophysiological experiments. The animal experiments comply with the "Principles of animal care," publication No. 86-23, revised 1985, of the National Institutes of Health, and also with the German law (approved by the Regierung von Oberbayern, 211-2531-31/97 and 211-2531-37/98). This study was supported by the DFG Ku $624 / 2-1$ and Ko 987/6-3.

\section{REFERENCES}

AITKIN LM. The auditory neurobiology of marsupials: a review. Hear. Res. 82:257-266, 1995.

Boege P, Jansen T. Pure-tone threshold estimation from extrapolated distortion product otoacoustic emission I/O-functions in normal and cochlear hearing loss ears. J. Acoust. Soc. Am. 111:1810-1818, 2002.

Brown AM, Kemp DT. Suppressibility of the $2 f_{1}-f_{2}$ stimulated acoustic emissions in gerbil and man. Hear. Res. 13:29-37, 1984.

CASSEDAY JH, Covey E. A neuroethological theory of the operation of the inferior colliculus. Brain Behav. Evol. 47:311-336, 1996.
Coles RB, Guppy A. Biophysical aspects of directional hearing in the tammar wallaby, Macropus eugenii. J. Exp. Biol. 121:371-394, 1986.

Dallos P. The active cochlea. J. Neurosci. 12:4575-4585, 1992.

Eisenberg JF, Gould E. The tenrecs: A study in mammalian behavior and evolution. Smiths. Contrib. Zool. 27:1-138, 1970.

Faulstich M, Kössl M. Neuronal response to cochlear distortion products in the anteroventral cochlear nucleus of the gerbil. J. Acoust. Soc. Am. 105:491-502, 1999.

FAulstich M, Kössl M. Evidence for multiple DPOAE components based upon group delay of the $2 \mathrm{f}(1)-\mathrm{f}(2)$ distortion in the gerbil. Hear. Res. 140:99-110, 2000.

Faulstich M, Kössl M, Reimer K. Analysis of non-linear cochlear mechanics in the marsupial Monodelphis domestica: ancestral and modern mammalian features. Hear. Res. 94:47-53, 1996.

Gorga MP, Neely ST, Ohlrich B, Hoover B, Redner J, Peters J. From laboratory to clinic: a large scale study of distortion product otoacoustic emissions in ears with normal hearing and ears with hearing loss. Ear Hear. 18:440-455, 1997.

Gould E. Evidence for echolocation in the Tenrecidae of Madagascar. Proc. Am. Phil. Soc. 109:352-360, 1965.

GuPPy A, Coles RB. Acoustical and neuronal aspects of hearing in the Australian gleaning bats, Macroderma gigas and Nyctophilus gouldi. J. Comp. Physiol. A 162:653-668, 1988.

Heffner RS, HefFner HE. Behavioral hearing range of the chinchilla. Hear. Res. 52:13-16, 1991.

Heffner RS, Koay G, Heffner HE. Audiograms of five species of rodents: implications for the evolution of hearing and the perception of pitch. Hear. Res. 157:138-152, 2001.

Jen H-S, Chen D. Directionality of sound pressure transformation at the pinna of echolocating bats. Hear. Res. 34:101-118, 1988.

Kimberley BP, BRown DK, EgGERMONT JJ. Measuring human cochlear traveling wave delay using distortion product emission phase responses. J. Acoust. Soc. Am. 94:1343-1350, 1993.

KoAy G, HefFner RS, HefFner HE. Hearing in a megachiropteran fruit bat (Rousettus aegyptiacus). J. Comp. Psychol. 112:371-382, 1998.

Kössl M. High-frequency distortions from the ears of two bat species, Megaderma lyra and Carollia perspicillata. Hear. Res. 602:156164, 1992.

Kössl M. Otoacoustic emissions from the cochlea of the "constant frequency" bats, Pteronotus parnellii and Rhinolophus rouxi. Hear. Res. 72:59-72, 1994.

Krubitzer L, KünZle H, KaAs JH. Organization of sensory cortex in a Madagascan insectivore, the tenrec (Echinops telfairi). J. Comp. Neurol. 379:399-414, 1997.

KüNZLE H. Care and breeding of the Madagascan hedgehog tenrec, Echinops telfairi, under laboratory conditions. Der Tierschutzbeauftragte 7:113-115, 1998.

KÜNZLE H, REHKÄMPER G. Distribution of cortical neurons projecting to dorsal column nuclear complex and spinal cord in the hedgehog tenrec, Echinops telfairi. Somatosens. Mot. Res. 9:185197, 1992.

Lasky RE, Soto AA, Luck ML, Laughlin NK. Otoacoustic emission, evoked potential, and behavioral auditory thresholds in the rhesus monkey (Macaco mulatta). Hear. Res. 136:35-43, 1999.

MANLey GA. Peripheral hearing mechanisms in reptiles and birds. Springer Verlag, Berlin, p 288, 1990.

MAnley GA, Köppl C, Johnston BM. Distortion-product Otoacoustic emissions in the bobtail lizard. I: General characteristics. J. Acoust. Soc. Am. 93:2820-2833, 1993.

Martin GK, Lonsbury-Martin BL, Probst R, Scheinin SA, Coats AC. Acoustic distortion products in rabbit ear canal. II. Sites of origin revealed by suppression contours and pure-tone exposures. Hear. Res. 28:191-208, 1987. 
Mills DM. Interpretation of distortion product Otoacoustic emission measurements. I. Two stimulus tones. J. Acoust. Soc. Am. 102:413-429, 1997.

Mills DM, Rubel EW. Development of distortion product emissions in the gerbil: "filter" response and signal delay. J. Acoust. Soc. Am. 101:395-411, 1997.

Mills DM, ShePherd RK. Distortion product otoacoustic emission and auditory brainstem responses in the echidna (Tachyglossus aculeatus). J. Assoc. Res. Otolaryngol. 2:130-146, 2001.

Muller M. The cochlear place-frequency map of the adult and developing Mongolian gerbil. Hear. Res 94:148-156, 1996.

Muller M, Wess FP, BRUNS V. Cochlear place-frequency map in the marsupial Monodelphis domestica. Hear. Res. 67:198-202, 1993.

Murphy WJ, Davis RR. The role of the chinchilla pinna and ear canal in electrophysiological measures of hearing thresholds. J. Acoust. Soc. Am. 103:1951-1956, 1998.

O'MAhoney CF, Kemp DT. Distortion product otoacoustic emission delay measurement in human ears. J. Acoust. Soc. Am. 97:37213735, 1995.

Popper AN, Fay RR. Evolution of the ear and hearing: issues and questions. Brain Behav. Evol. 50:213-221, 1997.

Probst R, Lonsbury-Martin BL, Martin GK. A review of otoacoustic emissions. J. Acoust. Soc. Am. 89:2027-2067, 1991.
ReIMER K. Hearing in the marsupial Monodelphis domestica as determined by auditory-evoked brainstem responses. Audiology 34:334-342, 1995.

RYAN AF. Hearing sensitivity of the mongolian gerbil, Meriones unguiculatis. J. Acoust. Soc. Am. 59:1222-1226, 1976.

Schuller G, Radtke-Schuller S, Betz M. A stereotaxic method for small animals using experimentally determined reference profiles. J. Neurosci. Methods 18:339-350, 1986.

Shera CA, Guinan Jr JJ. Evoked otoacoustic emissions arise by two fundamentally different mechanisms: A taxonomy for mammalian OAEs. J. Acoust. Soc. Am. 105:782-798, 1998.

Springer MS, Cleven GC, Madsen O, de Jong WW, Waddell VG, Amrine HM, Stanhope MJ. Endemic African mammals shake the phylogenetic tree. Nature 388:61-64, 1997.

Stephan H, Baron G, Frahm HD. Comparative Brain Research in Mammals: Insectivora. Springer Verlag, New York, 1991.

Whitehead ML, Lonsbury-Martin BL, Martin GK, McCoy MJ. Otoacoustic emissions: animal models and clinical observations. In: Van de Water TR, Popper AN, Fay RR (Eds.) Clinical aspects of hearing. Springer-Verlag, New York, pp 199-257, 1996.

Willard FH, Martin GF. The auditory brainstem nuclei and some of their projections to the inferior colliculus in the North American opossum. Neuroscience 10:1203-1232, 1983. 